\title{
Correlation between Liver Metastases and the Level of PRL-3 mRNA Expression in Patients with Primary Colorectal Cancer
}

\author{
Nam Won Kim, Chong Woo Chu', Tae Sung Ahn, Chang Jin $\mathrm{Kim}^{2}$, Dong Jun Jung ${ }^{2}$, Myoung Won Son, \\ Sang Ho Bae, Moon Soo Lee, Chang Ho Kim, Moo Jun Baek \\ Department of Surgery, Soonchunhyang University College of Medicine, Cheonan; ${ }^{1}$ Department of Surgery, Pusan National University \\ Yangsan Hospital, Yangsan; ${ }^{2}$ Department of Pathology, Soonchunhyang University College of Medicine, Cheonan, Korea
}

Purpose: Phosphatase of regenerating liver-3 (PRL-3) has been associated with metastasis promotion. However, clinical applications of this association have not yet been clearly demonstrated. In this study, we evaluated the relation of PRL-3 mRNA level in primary colorectal cancer to the corresponding stage and to other clinicopathologic factors.

Methods: Two hundred forty-five patients with histologically-proven colorectal cancer underwent surgery between January 2004 and December 2006. RNA was extracted and cDNA was prepared by using reverse transcription. Quantification of PRL-3 was done using a real-time polymerase chain reaction.

Results: Eighty-six cases with well-preserved specimens were enrolled: 53 males and 33 females. The mean age was 63.4 years. According to tumour node metastasis (TNM) stage of the American Joint Committee on Cancer (AJCC), stage I was 11 cases, stage II was 38 cases, stage III was 23 cases, and stage IV was 14 cases. Among stage IV cases, one case was combined with liver and lung metastases, and one case was combined with liver metastases and peritoneal dissemination. The remaining stage IV patients were combined with only liver metastases. There was a significant correlation in PRL-3 mRNA expression between primary colorectal cancer and corresponding tumor stage. PRL-3 mRNA expression was increased in the liver metastases cases. Lymphatic and vascular invasion were significantly related with PRL-3 mRNA levels.

Conclusion: Advanced stage prediction may be obtained by measuring the level of PRL-3 mRNA expression in primary colorectal cancer. Especially, the risk of liver metastases may be predicted by measuring the level of PRL-3 mRNA expression in primary colorectal cancer. Further study is required to confirm these preliminary results.

Keywords: PTP4A3 protein, human; Real-time polymerase chain reaction; Colorectal neoplasms; Neoplasm Metastasis

\section{INTRODUCTION}

Metastasis is a major cause of colorectal cancer death. Most of the patients die of metastatic disease rather than the primary colorectal cancer. Through a number of studies, genetic and biochemical

Received: July 20, 2011 Accepted: August 26, 2011

Correspondence to: Moo-Jun Baek, M.D.

Department of Surgery, Soonchunhyang University College of Medicine,

23-20 Bongmyung-dong, Dongnam-gu, Cheonan 330-721, Korea

Tel: +82-41-570-3633, Fax: +82-41-571-0129

E-mail: ssurge@sch.ac.kr

(C) 2011 The Korean Society of Coloproctology

This is an open-access article distributed under the terms of the Creative Commons Attribution NonCommercial License (http://creativecommons.org/licenses/by-nc/3.0) which permits unrestricted noncommercial use, distribution, and reproduction in any medium, provided the original work is properly cited. mechanisms for the metastases of malignant tumors, including colorectal cancer, has been found, but achieving a clear understanding of the genes or materials causing metastasis is difficult.

Protein tyrosine phosphatase (PTP) is a material playing an essential role in regulating various proteins involved in physiological and other processes that cause diseases [1]. Unlike phosphorylation of serine or threonine, phosphorylation of the protein tyrosine is found only in eukaryotes. Phosphorylation of the protein tyrosine is important for the functions of eukaryotes, such as cell proliferation, cell differentiation, metabolism, intercellular communication, cell movement, genetic transcription, immune response and ion channeling [2]. Moreover, abnormal phosphorylation of tyrosine is related with various diseases, including cancer, diabetes, rheumatoid arthritis and hypertenstion. Phosphorylation of tyrosine is a reversible process and is regulated by the pro- 
tein tyrosine kinase (PTK), which is involved in the phosphorylation of two complimentary enzymes, and the PTP, which is the enzyme involved in dephosphorylation. Both PTK and PTP produce large protein families composed of many different enzymes $[3,4]$. PTP can amplify or reduce intracellular signal transduction and is important for the functions of eukaryotes [5]. If the functions of PTP are not well regulated, the development and progression of various diseases are promoted [6]. Among the proteins produced by PTP, phosphatase of regenerating liver (PRL) has 3 subcategories, PRL-1, PRL-2, and PRL-3, each of which has a size of roughly $20 \mathrm{KDa}$ and a homology in amino acid structure of more than $75 \%[6,7]$. PRL-1 is known to be an immediate early gene controlling regeneration of the liver [8]. Excessive expression of PRL-1 or PRL-2 is known to promote tumor growth in nude mice [7].

PTP4A3, which is also known to be a phosphatase of regenerating liver-3, is a member of the PTP super family and is a gene related to progress, invasion and metastasis of malignant tumors [911]. PRL-3 is a phosphatase having small molecular weight with a size of $22 \mathrm{KDa}$. Among human adult normal tissues, PRL-3 mRNA is mainly expressed in skeletal muscles or the heart and is expressed at low levels in the pancreas, spleen, lung and testis $[10,12]$. The physiological functions of PRL-3 in those tissues have not yet been clarified.

The functions of members of the PRL family are understood to play important roles in the processes of initiation and development of tumors such as cancer cell invasion and migration, neovascularization of tumors, and metastasis [9, 13-15]. A unique excessive expression of PRL-3 was initially observed in primary colorectal cancer tissues [13]. Later, excessive expression of PRL-3 was verified as promoting cellular migration, invasion and metastasis in Chinese hamster ovary cells [9]. In vivo and in vitro experiments confirmed that PRL-3 induced endothelial cells to initiate angiogenesis of tumors and then to promote metastasis of the tumors [15]. Additionally, increased expression of PRL-3 has been reported to be related to the progression and metastasis of cancer in various tumors, such as breast cancer [16], ovarian cancer [17], and stomach cancer.

In our previous study [18] on protein expression of PRL-3 in primary colorectal cancer tissues, we reported a higher level of protein expression of PRL-3 in primary colorectal cancer tissues with hepatic metastasis than in those without metastasis. In the present study, we tried to analyze the expression of PRL-3 mRNA in primary colorectal cancer tissues and its effects on the progression of colorectal cancer, especially its relation with the existence of hepatic metastasis, by using a real-time reverse transcription-polymerase chain reaction (RT-PCR) to quantitatively analyze PRL-3 mRNA expression. We compared the results of RT-PCR with various clinicopathological factors to determine if the existence of hepatic metastasis could be predicted; in particular, we used PRL-3 expression in primary colorectal cancer tissues to find relations between hepatic metastasis and the clinicopathological factors.

\section{METHODS}

\section{Patients}

A total of 86 patients who visited Soonchunhyang University Cheonan Hospital, were histologically diagnosed with colorectal cancer and then underwent surgery between the period of January 2004 and December 2006 were enrolled in the present study. No patient received chemotherapy or radiation therapy prior to surgery, which was radical surgery. Excised tissues were immediately cooled by using liquid nitrogen and were kept frozen in a freezer at $-80^{\circ} \mathrm{C}$ before extracting RNA. For staging, we followed the tumour node metastasis (TNM) classification of malignant tumors of the American Joint Committee on Cancer (AJCC), 7th edition, revised in 2010 .

\section{Methodology}

\section{RNA extraction and reverse transcription}

The RNA of the colorectal cancer cells kept at $-80^{\circ} \mathrm{C}$ was extracted using Totally RNA ${ }^{\mathrm{TM}}$ (Ambion Inc., Austin, TX, USA) and was purified using RNeasy to prevent the extracted RNA from being mixed with DNA. The integrity of the extracted RNA was determined by using agar electrophoresis to analyze intact 18S/28S rRNA bands. RNA was reverse transcribed to cDNA, together with oligo (dT) primer, by using a Power cDNA synthesis kit (Intron, Seongnam, Korea). One $\mu \mathrm{g}$ of RNA was cultured in $20 \mu \mathrm{L}$ of solute $(1 \mu \mathrm{L}$ of RNase inhibitor, $4 \mu \mathrm{L}$ of $5 \mathrm{X}$ RT buffer, $2 \mu \mathrm{L}$ of dNTP, $2 \mu \mathrm{L}$ of DTT, and $0.5 \mu \mathrm{L}$ of AMV RT enzyme) at $42^{\circ} \mathrm{C}$ for 60 minutes. The RT reaction was conducted at $70^{\circ} \mathrm{C}$ for over 5 minutes.

\section{Quantitative real-time detection of PCR}

Quantitative real-time RT-PCR analyses were conducted using the ABI PRISM 7000 Sequence Detection System and the QuantiTect SYBR Green RT-PCR kit (Qiagen, Seoul, Korea). As a primer for amplifying the products of the reaction, forward 5'-GGG ACT TCT CAG GTC GTG TC-3' and 5'-AGC CCC GTA CTT CTT CAG GT-3' were prepared. As a primer for amplifying $\beta$-actin with a control material detecting RNA, forward $5^{\prime}$-CCA CGA AAC

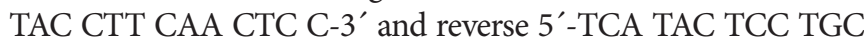
TGC TTG CTG ATC C-3' were used. According to the manufacturer's instruction, master-mix $(50 \mu \mathrm{L})$ was prepared with the combinations of $2 \times$ QuantiTect SYBR Green RT-PCR Master Mix (25 $\mu \mathrm{L})$, each primer at $0.5 \mu \mathrm{mol} / \mathrm{L}$, QuantiTect RT Mix $(0.5 \mu \mathrm{L})$, total RNA $(10 \mu \mathrm{L}, 10 \mathrm{ng})$ and RNase-free water. Amplification was conducted by repeating the process 40 times, which involved an initial 30 -minute reaction at $50^{\circ} \mathrm{C}$, followed by 15 -minute denaturation at $95^{\circ} \mathrm{C}, 15$-minute denaturation at $94^{\circ} \mathrm{C}, 30$-minute annealing at $60^{\circ} \mathrm{C}$, and 1 -minute elongation at $72^{\circ} \mathrm{C}$. All tests were repeated 2 times.

\section{Statistical analysis}

The level of PRL-3 mRNA expression, its effects on lymph-node 
invasion, and its relation with neural invasion, lymphatic invasion, and vascular invasion were examined using the Mann-Whitney test in the SPSS ver. 12.0 (SPSS Inc., Chicago, IL, USA) program. Relations with staging and differentiation level of primary colorectal cancer were analyzed using the ANOVA test and were evaluated as being statistically significant when the P-value was less than 0.05 .

\section{RESULTS}

\section{Patients characteristics}

The 86 subjects included 53 males and 33 females, with ages ranging from 34 to 92 years and a mean age of 64.35 years. According to the TNM classification of malignant tumors of the AJCC, 11 subjects were in stage I (12.8\%), 38 were in stage II (44.2\%), 23 were in stage III (26.7\%), and 14 were in stage IV (16.3\%). In terms of cell differentiation, moderately differentiated cancer had the greatest number of 67 (77.9\%). Out of 14 cases in stage IV, 1 case had both liver and lung metastases, another case had both liver metastasis and intraperitoneal dissemination, and the remaining cases had liver metastases only (Table 1).

Table 1. Clinicopathological variables of patients

\begin{tabular}{lc}
\hline Characteristics & Frequency (\%) \\
\hline Age (yr), mean \pm SD & $64.87 \pm 11.74$ \\
Gender & $53(61.6)$ \\
Male & $33(38.4)$ \\
Female & \\
Histology & $6(7.0)$ \\
Well differentiated & $67(77.9)$ \\
Moderately differentiated & $8(9.3)$ \\
Poorly & $5(5.8)$ \\
Unknown & \\
Lymphatic invasion & $70(81.4)$ \\
No & $16(18.6)$ \\
Yes & \\
Vascular invasion & $71(82.6)$ \\
No & $15(17.4)$ \\
Yes & \\
Neural invasion & $78(90.7)$ \\
No & $8(9.3)$ \\
Yes & \\
Stage & $11(12.8)$ \\
I & $38(44.2)$ \\
\hline IV & $23(26.7)$ \\
\hline
\end{tabular}

\section{Relation between the level of mRNA expression in primary} colorectal cancer tissues and clinicopathological factors The level of expression of PRL-3 mRNA in primary colorectal cancer tissues and various clinicopathological factors in the colorectal cancer patients were compared. Stage, lymph-node invasion, vascular invasion, lymphatic invasion, neural invasion, cell differentiation and survival rates were compared, Among these, amount of PRL-3 mRNA was significantly higher in cases involving lymphatic invasion cancer cells $(\mathrm{P}=0.021)$ and vascular invasion $(\mathrm{P}=0.001)$ by cancer cells. In terms of stage, the larger the amount of PRL-3 mRNA expression was, the more advanced the stage was $(\mathrm{P}<0.001)$ (Fig. 1). However, in terms of neural invasion $(\mathrm{P}=0.563)$ and cancer cell differentiation ( $\mathrm{P}=0.887$ ), no significant differences were found in the amount of PRL-3 mRNA (Table 2). Additionally, no significant difference was found in terms of the age or the sex of the patients.

\section{Relation between the level of mRNA expression in primary colorectal cancer tissues and hepatic metastasis of colorectal cancer}

The level of $P R L-3$ mRNA expression in primary colorectal cancer tissues and the presence of hepatic metastasis of colorectal cancer were compared, and an increase in the amount of PRL-3 mRNA expression was confirmed to be related to an increase in hepatic metastasis of colorectal cancer $(\mathrm{P}=0.05)$ (Fig. 2).

\section{DISCUSSION}

Most deaths from colorectal cancer are caused by metastasis, not by the primary colorectal cancer itself. However, the molecular

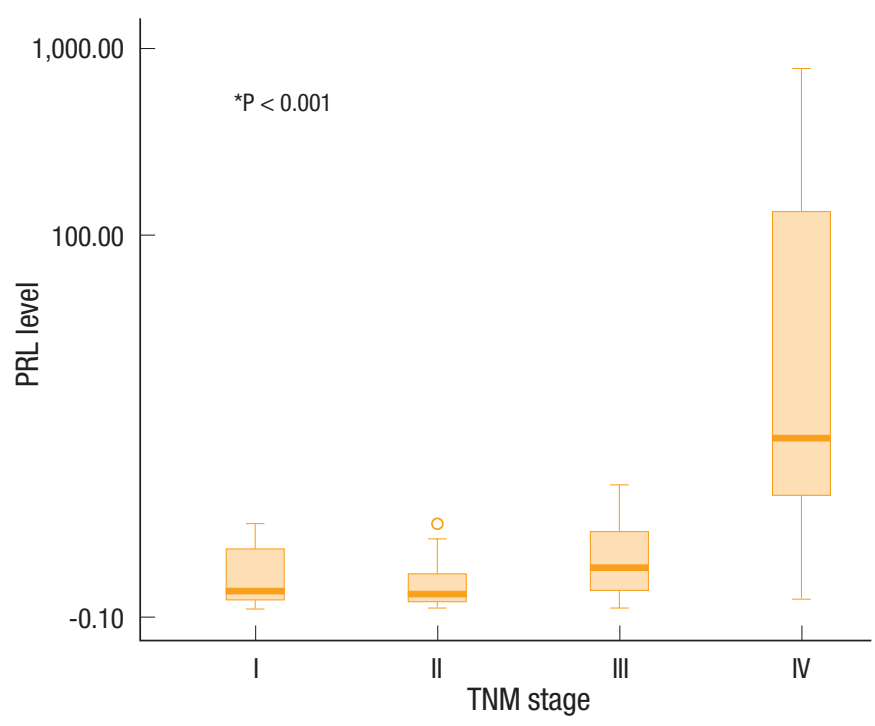

Fig. 1. Box plots of the phosphatase of regenerating liver (PRL) level of colorectal cancer according to tumour node metastasis (TNM) stage. The $\mathrm{Y}$ axis is represented by a logarithmic scale. 
Table 2. Mean phosphatase of regenerating liver (PRL) gene expression levels in colorectal carcinomas

\begin{tabular}{|c|c|c|c|}
\hline & No. & PRL expression levels & P-value \\
\hline Gender & & & 0.546 \\
\hline Male & 53 & $18.18 \pm 108.71$ & \\
\hline Female & 33 & $34.61 \pm 141.14$ & \\
\hline Histology & & & 0.887 \\
\hline Well differentiated & 6 & $1.52 \pm 2.13$ & \\
\hline Moderately differentiated & 67 & $29.84 \pm 131.17$ & \\
\hline Poorly differentiated & 8 & $0.83 \pm 0.79$ & \\
\hline Unknown & 5 & $24.87 \pm 125.07$ & \\
\hline Lymphatic invasion & & & 0.021 \\
\hline+ & 70 & $26.02 \pm 133.74$ & \\
\hline- & 16 & $17.79 \pm 38.67$ & \\
\hline Vascular invasion & & & 0.001 \\
\hline+ & 71 & $26.94 \pm 127.54$ & \\
\hline- & 15 & $0.611 \pm 0.50$ & \\
\hline Neural invasion & & & 0.563 \\
\hline+ & 78 & $25.60 \pm 132.83$ & \\
\hline- & 8 & $19.21 \pm 39.61$ & \\
\hline Stage & & & $<0.001$ \\
\hline I & 11 & $0.68 \pm 0.70$ & \\
\hline$\|$ & 38 & $0.39 \pm 0.41$ & \\
\hline III & 23 & $1.00 \pm 0.99$ & \\
\hline IV & 14 & $147.20 \pm 278.14$ & \\
\hline Liver metastasis & & & 0.05 \\
\hline No & 72 & $0.63 \pm 0.73$ & \\
\hline Yes & 14 & $147.20 \pm 278.14$ & \\
\hline
\end{tabular}

Values are presented as mean \pm SD.

biological mechanism regulating metastasis of colorectal cancer is still unknown. As reported by Vogelstein for the first time, various tumor suppressor genes and oncogenes are involved in the development process from a normal colorectal mucosa to an early adenoma, an advanced adenoma and finally malignant colorectal cancer, but the genes involved in metastasis after the development of a malignant tumor have not yet been identified.

PTPs play a fundamental role in regulating diverse proteins that participate essentially in every aspect of physiological and pathogenic cellular processes [1]. The PRL phosphatases (PRL-1, -2, and -3) are three closely related intracellular enzymes that possess PTP active sites and are believed to play key roles in cellular transformation. Like the PTP active site signature sequence CX5R, they are molecules having a unique $\mathrm{COOH}$-terminal prenylation motif [19]. These PTPs have a size of roughly $20 \mathrm{KDa}$ and share a $75 \%$ amino acid sequence similarity [7]. According to various research, PRL-3 plays an important role during invasion and metastasis of tumors

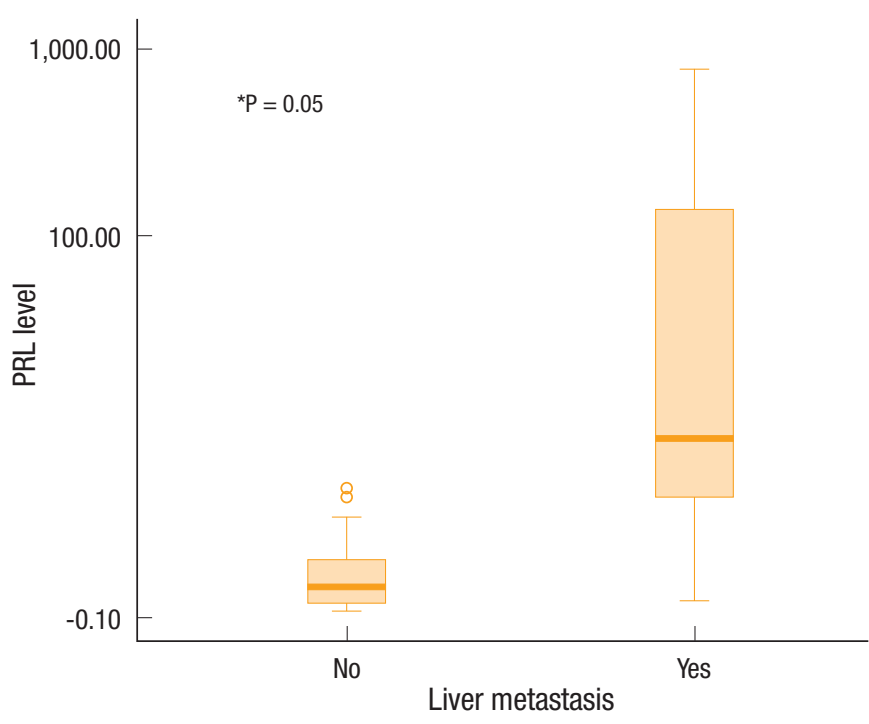

Fig. 2. Box plot of the phosphatase of regenerating liver (PRL) level of colorectal cancer according to hepatic metastasis. The $\mathrm{Y}$ axis is represented by a logarithmic scale.

$[9,10,13,17]$. However, the role of PRL-3 in the carcinogenesis and progression of colorectal cancer has not yet been clarified.

According to the present study, tissues from patients having colorectal cancer with hepatic metastasis were confirmed to show higher levels of phosphatase PRL-3 than tissues from patients having colorectal cancer without metastasis. Phosphatase PRL-3 was also confirmed to have relations with aggressive factors of cancer, such as lymphatic or vascular invasion, and with the stage of colorectal cancer.

When PRL-3 is excessively expressed in a human malignant tumor, the PRL-3 gene is located in the human chromosome 8q. In 3 of 12 metastases examined, multiple copies of the PRL-3 gene were found within a small amplicon located at chromosome 8q24.3. Moreover, extra copies of this region are often found in advanced stages of different tumor types. Thus, PRL-3 genes may be assumed to play an important role in metastasis of various malignant tumors, including colorectal cancer [13, 20, 21]. Further, according to recent studies, PRL-3 is related with PI3K-Akt, STAT3-Rho, $\mathrm{Csk} / \mathrm{Src}$ and $\mathrm{p} 53$ signal transduction pathways [22-25]. Upon verifying the signal transduction pathway in the future, extensive information on molecular biology regarding the effect of PRL-3 on the progression of cancer, specifically on hepatic metastasis, may be obtained.

PRL-3 has been understood as being a gene related with metastasis of colorectal cancer. In the present study, we wanted to confirm the relation between $\mathrm{mRNA}$ expression of PRL-3 and colorectal cancer with hepatic metastasis, specifically primary colorectal cancer with hepatic metastasis. We confirmed that the expression level of PRL-3 mRNA was related with the progression of colorectal cancer. More specifically, we confirmed that excessive expression of PRL-3 mRNA in tissues of the primary colorectal cancer 
was related with hepatic metastasis of colorectal cancer.

According to initial research on the expression of PRL-3 in colorectal cancer, this enzyme is not expressed in the early stage of tumor development, but is expressed only in metastasized cancer cells [14], so PRL-3 is found neither in the normal mucosa nor in colorectal cancer without metastasis. Interpretations of the effects of PRL-3 on cell growth do not agree among various reports. According to a study, for example, ectopic expression of PRL-3 promotes cell proliferation $[7,26]$, but according to another study using the DLD-1 colon cancer cell line, temporary down regulation of PRL-3 by small interfering RNA does not seemingly affect cell proliferation [27]. Polato et al. [17] reported that when PRL-3 siRNA was incorporated into the HCT-116 colon cancer cell line, cell growth was not affected, even though a low level of PRL-3 was expressed.

Various researchers expect PRL-3 expression to be required for metastasis of a tumor in its early stage, for which lymphatic and vascular invasion has occurred [27]. Other researchers found that PRL-3 might be used as a tumor marker for metastasis of colorectal cancer through immunostaining and that expression of PRL-3 might adversely affect the prognosis for a colorectal cancer patient [28]. Recent studies have reported many cases with unfavorable effects of expression of PRL-3 on breast cancer, ovarian cancer and stomach cancer $[17,29]$.

According to the recent study of Radke et al. [16], the diseasefree survival rate of lymph-node-metastasized breast cancer patients with excessive expression of PRL-3 was significantly lower than that of patients without PRL-3 expression. However, the same authors' previous study showed no difference in survival rates when expression of PRL-3 using immunostaining was compared with the cases of colorectal cancer patients [18].

In our previous study, we confirmed the expression of PRL-3 in primary colorectal cancer tissues by using immunohistochemical staining, and we compared the results with factors such as age, sex, size of tumor, cancer cell differentiation, depth of invasion, microscopic findings of lymphatic metastasis, hematogenous metastasis, number of lymph-node metastases, post-operative tumor stages and survival rates through a retrospective study to find correlations between those factors and PRL-3 expression. As a result, PRL-3 expression showed a significant relation with lymphatic invasion and $T$ stage of the tumor [18]. In addition, in 15 cases out of a total of 16 hepatic metastasis cases, positive expression of PRL-3 showed a correlation with hepatic metastasis. Even though we could not obtain exact survival rates in the present study, considering the adverse effects of correlations among PRL-3 and clinicopathological factors on the survival rates, PRL-3 expression in primary colorectal cancer is evidently related with a worse prognosis for colorectal patients.

According to our study, PRL-3 may be an important factor contributing to the invasion and metastasis of colorectal cancer cells. A high level of expression of PRL-3 mRNA was found in the primary colorectal cancer tissues of a colorectal cancer patient with hepatic metastasis, and expression of PRL-3 mRNA is understood to affect lymphatic and vascular invasion and stages of colorectal cancer. Thus, PRL-3 plays an important role in the progression, invasion and metastasis of primary colorectal cancer. Consequently, PRL-3 may possibly be used as a marker for measuring the progression and invasion of colorectal cancer. In addition, PRL-3 may also be used as a target for developing methods of treating metastatic colorectal cancer, which is still difficult to cure.

\section{CONFLICT OF INTEREST}

No potential conflict of interest relevant to this article was reported.

\section{REFERENCES}

1. Zhang ZY, Zhou B, Xie L. Modulation of protein kinase signaling by protein phosphatases and inhibitors. Pharmacol Ther 2002; 93:307-17.

2. Hunter T. Signaling: 2000 and beyond. Cell 2000;100:113-27.

3. Li L, Dixon JE. Form, function, and regulation of protein tyrosine phosphatases and their involvement in human diseases. Semin Immunol 2000;12:75-84.

4. Neel BG, Tonks NK. Protein tyrosine phosphatases in signal transduction. Curr Opin Cell Biol 1997;9:193-204.

5. Van Vactor D, O'Reilly AM, Neel BG. Genetic analysis of protein tyrosine phosphatases. Curr Opin Genet Dev 1998;8:112-26.

6. Zhang ZY. Protein tyrosine phosphatases: prospects for therapeutics. Curr Opin Chem Biol 2001;5:416-23.

7. Diamond RH, Cressman DE, Laz TM, Abrams CS, Taub R. PRL-1, a unique nuclear protein tyrosine phosphatase, affects cell growth. Mol Cell Biol 1994;14:3752-62.

8. Mohn KL, Laz TM, Hsu JC, Melby AE, Bravo R, Taub R. The immediate-early growth response in regenerating liver and insulinstimulated H-35 cells: comparison with serum-stimulated 3T3 cells and identification of 41 novel immediate-early genes. Mol Cell Biol 1991;11:381-90.

9. Wu X, Zeng H, Zhang X, Zhao Y, Sha H, Ge X, et al. Phosphatase of regenerating liver- 3 promotes motility and metastasis of mouse melanoma cells. Am J Pathol 2004;164:2039-54.

10. Li YH, Hu CF, Shao Q, Huang MY, Hou JH, Xie D, et al. Elevated expressions of survivin and VEGF protein are strong independent predictors of survival in advanced nasopharyngeal carcinoma. J Transl Med 2008;6:1.

11. Takano Y, Kato Y, Masuda M, Ohshima Y, Okayasu I. Cyclin D2, but not cyclin D1, overexpression closely correlates with gastric cancer progression and prognosis. J Pathol 1999;189:194-200.

12. Matter WF, Estridge T, Zhang C, Belagaje R, Stancato L, Dixon J, et al. Role of PRL-3, a human muscle-specific tyrosine phosphatase, in angiotensin-II signaling. Biochem Biophys Res Commun 2001;283:1061-8.

13. Saha S, Bardelli A, Buckhaults P, Velculescu VE, Rago C, St Croix $\mathrm{B}$, et al. A phosphatase associated with metastasis of colorectal cancer. Science 2001;294:1343-6. 
14. Bardelli A, Saha S, Sager JA, Romans KE, Xin B, Markowitz SD, et al. PRL-3 expression in metastatic cancers. Clin Cancer Res 2003; 9:5607-15.

15. Guo K, Li J, Wang H, Osato M, Tang JP, Quah SY, et al. PRL-3 initiates tumor angiogenesis by recruiting endothelial cells in vitro and in vivo. Cancer Res 2006;66:9625-35.

16. Radke I, Gotte M, Kersting C, Mattsson B, Kiesel L, Wulfing P. Expression and prognostic impact of the protein tyrosine phosphatases PRL-1, PRL-2, and PRL-3 in breast cancer. Br J Cancer 2006; 95:347-54.

17. Polato F, Codegoni A, Fruscio R, Perego P, Mangioni C, Saha S, et al. PRL-3 phosphatase is implicated in ovarian cancer growth. Clin Cancer Res 2005;11(19 Pt 1):6835-9.

18. Lee HJ, Chu CW, Baek MJ, Shin EJ, Kim HC, Cho GS, et al. PRL-3 expression in primary colorectal cancer. J Korean Soc Coloproctol 2007;23:497-502.

19. Zeng Q, Hong W, Tan YH. Mouse PRL-2 and PRL-3, two potentially prenylated protein tyrosine phosphatases homologous to PRL-1. Biochem Biophys Res Commun 1998;244:421-7.

20. Alers JC, Krijtenburg PJ, Rosenberg C, Hop WC, Verkerk AM, Schroder $\mathrm{FH}$, et al. Interphase cytogenetics of prostatic tumor progression: specific chromosomal abnormalities are involved in metastasis to the bone. Lab Invest 1997;77:437-48.

21. Paredes-Zaglul A, Kang JJ, Essig YP, Mao W, Irby R, Wloch M, et al. Analysis of colorectal cancer by comparative genomic hybridization: evidence for induction of the metastatic phenotype by loss of tumor suppressor genes. Clin Cancer Res 1998;4:879-86.

22. Fiordalisi JJ, Keller PJ, Cox AD. PRL tyrosine phosphatases regu- late rho family GTPases to promote invasion and motility. Cancer Res 2006;66:3153-61.

23. Liang F, Liang J, Wang WQ, Sun JP, Udho E, Zhang ZY. PRL3 promotes cell invasion and proliferation by down-regulation of Csk leading to Src activation. J Biol Chem 2007;282:5413-9.

24. Wang H, Quah SY, Dong JM, Manser E, Tang JP, Zeng Q. PRL-3 down-regulates PTEN expression and signals through PI3K to promote epithelial-mesenchymal transition. Cancer Res 2007;67: 2922-6.

25. Basak S, Jacobs SB, Krieg AJ, Pathak N, Zeng Q, Kaldis P, et al. The metastasis-associated gene Prl-3 is a p53 target involved in cellcycle regulation. Mol Cell 2008;30:303-14.

26. Werner SR, Lee PA, DeCamp MW, Crowell DN, Randall SK, Crowell PL. Enhanced cell cycle progression and down regulation of p21(Cip1/Waf1) by PRL tyrosine phosphatases. Cancer Lett 2003; 202:201-11.

27. Kato H, Semba S, Miskad UA, Seo Y, Kasuga M, Yokozaki H. High expression of PRL-3 promotes cancer cell motility and liver metastasis in human colorectal cancer: a predictive molecular marker of metachronous liver and lung metastases. Clin Cancer Res 2004; 10:7318-28.

28. Peng L, Ning J, Meng L, Shou C. The association of the expression level of protein tyrosine phosphatase PRL-3 protein with liver metastasis and prognosis of patients with colorectal cancer. J Cancer Res Clin Oncol 2004;130:521-6.

29. Song LB, Yan J, Jian SW, Zhang L, Li MZ, Li D, et al. Molecular mechanisms of tumorgenesis and metastasis in nasopharyngeal carcinoma cell sublines. Ai Zheng 2002;21:158-62. 